\title{
HIGH-EFFICIENCY TARGET-ION SOURCES FOR RIB GENERATION
}

\author{
G. D. Alton, Physics Division, Oak Ridge National Laboratory \\ Oak Ridge, Tennessee 37831-6368
}

\begin{abstract}
In this report, emphasis is placed on issues related to selection and design of high-temperature, ion sources that have demonstrated the high ionization efficiency, species versatility, and operational reliability required at ISOL based radioactive ion beam facilities. In designing sources for ISOL applications, careful attention must be given to the following: selecting the most appropriate materials of construction, coupling of the vapor transport system, ion optics, operational parameters, thermal transport properties, emittances, ionization efficiencies and engineering details for safe handling in the high-level radioactivity radiation fields incumbent at such facilities. Included in the article are descriptions and performance data for high temperature, positive (electron-impact and surface-ionization) and negative (kinetic-ejection and surface-ionization) ion sources, as well as, low temperature, batch-mode negative-ion sources, developed for processing long-lived isotopes, that have been principal contributors to recent successes held at the Holifield Radioactive Ion Beam Facility.
\end{abstract}

\section{INTRODUCTION}

Many of the nuclear reactions important in nuclear structure and nuclear astrophysics are inaccessible to experimental study using stable/stable beam/target combinations and therefore, can only be studied with accelerated radioactive ion beams (RIBs). The availability of RIBs for these purposes offers unique opportunities to further our knowledge about the structure of the nucleus, the stellar processes that power the universe, and the nucleosynthesis processes responsible for heavy element formation. As a consequence, world-wide interest has motivated the construction of facilities dedicated to research with such beams, including the Holifield Radioactive Ion Beam Facility (HRIBF), Oak Ridge National Laboratory (ORNL) [1]. These facilities either rely on projectile fragmentation to form short-lived species by break-up of energetic beams of heavy ions as they pass through a thin production target or on the Isotope-Separator-On-Line (ISOL) technique, of which the HRIBF is an example. In the latter type facility, radioactive nuclei are produced by irradiating solid or liquid target materials with light-ion beams from a driver accelerator; consequently, short-lived species, must be diffused from the matrix of target materials, effusively transported to an ion source, ionized, extracted, and accelerated in a time commensurate with their lifetimes.

The HRIBF is a fully implemented, first-generation, Isotope-Separator-On-Line (ISOL) based RIB facility dedicated to the production, generation, and post- acceleration of short-lived radioactive ion beams (RIBs) with the 25-MV tandem electrostatic accelerator to energies sufficiently high for nuclear physics and nuclear astrophysics research. The HRIBF utilizes light-ion beams $\left(p, d,{ }^{3} \mathrm{He}^{++}\right.$and $\left.{ }^{4} \mathrm{He}^{++}\right)$from the Oak Ridge Isochronous Cyclotron (ORIC) for the production of short-lived species within the matrices of small dimensioned target materials. Following diffusion release, these species are transported to an ion source, negatively ionized and then injected from a high voltage platform into the 25-MV tandem electrostatic accelerator where light-ion beams are accelerated to energies between 0.1 and $10 \mathrm{MeV} / \mathrm{u}$ and intermediate mass beams (e.g., $\mathrm{M} \leq$ $100 \mathrm{u})$ to energies up to $5 \mathrm{MeV} / \mathrm{u}$. Since the tandem relies on the injection of negative-ion beams, RIBs of this polarity must be directly generated in either Cs-sputter or negative-surface ionization sources or by conversion of initially positive-ion beams through charge exchange.

The integrated times required for the diffusion and effusive-flow processes determine the minimum lifetime of species that can be processed and consequently, the number of species that can be delivered to the research station. Thus, delay times attributable to diffusion and effusive processes must be minimized in relation to the lifetimes of the species of interest and highly efficient ion sources must be chosen in order to deliver useful intensities to the research station. Consequently, major thrusts of the research and development programs at the HRIBF have been directed toward reducing such delays by developing small dimensioned, highly permeable targets, high efficiency ion sources and optimized vapor transport systems. Many ion source developments have been made over the past few decades for ISOL applications. (For information on relatively recent developments in on-line mass separators and target-ion sources associated with their use, see, e.g., Refs. 2 and 3.) In the present article, sources will be described that have been principal contributors to recent successes held at the HRIBF.

\section{HRIBF ION SOURCES FOR RIB GENERATION}

ISOL ion source development continues to be driven by needs for sources with improved chemical selectivity, high duty factors and more universal species capabilities. Ideally, ion sources for RIB generation must exhibit the following properties: high efficiency, low energy spread, chemical selectivity, operation at very high and controllable temperatures, flexibility for adaptation to other ranges of temperature and modes of operation, high reliability, long lifetime, and stable electrical and 
mechanical properties. Of paramount importance, is the fact that the source must be mechanically designed for safe and expedient installation/removal from the production target station to permit interchange of sources and target materials and repair of failed components. We have adapted the engineering principles embodied in the source modules used at CERN-ISOLDE for remote and safe handling of their target/ion source systems [see, e.g., Ref. 4]. Each of the HRIBF target/ion sources is designed to fit into a modular vacuum chamber that can be robotically removed for servicing or storage by disconnection from, or reconnection to the beam transport system. An isometric drawing of a high temperature ion source is shown in Fig. 1. All high temperature sources, mounted in the vacuum housing, are similar in size and geometry and only the aspects of the ionization region of the sources differ. HRIBF ion sources, in their usual construction are made of $\mathrm{Ta}$, but other refractory materials may be preferable for processing particular species. For example, Re or Ir liners can be very effective in reducing effusive flow times from targets to the ion source for electronegative elements [5,6]. The effusiveflow times can be also be reduced by optimally coupling (increasing the vacuum conductivity) the target to the ion source. The target material reservoirs of all HRIBF high temperature sources can be independently heated to controllable temperatures exceeding $2000{ }^{\circ} \mathrm{C}$ by passing a current through a triple-pass Ta heater surrounding the reservoir. The transport tube between the target reservoir and ionization chamber of the source can also be independently heated to these temperatures, by passing a current through the transport tube. The temperature at the exit end of the transport tube must be controlled carefully as required for optimizing the ionization process or maintaining stability of operation. For example, the exit of the transport tube serves as the electron emitter (cathode) for the Electron Beam Plasma Ion Source, described below, and must be continuously operated at thermionic emission temperatures $\left(\sim 2150{ }^{\circ} \mathrm{C}\right)$. Positivesurface ionization sources that utilize specific metal ionizers must be operated in a narrow temperature range slightly above their respective critical temperatures (e.g., $\sim 1200{ }^{\circ} \mathrm{C}$ for $\mathrm{W}$ ). In cases where the ionizer is made of a compound (e.g., negative-surface ionization sources equipped with $\mathrm{LaB}_{6}$ ionizers), the end of the transport tube must be kept below the thermal dissociation temperature of the ionizer material. Therefore, in order to minimize delay times due to diffusion and thereby maximize RIB intensities, careful attention must be given in selecting the most appropriate and most refractory production target materials and in engineering their design so that they meet the dimensional and high permeability criteria for fast diffusion release and transport through the target material $[6,7]$. Equal attention must be paid to the selection of materials of construction for the target material reservoir and vapor transport system while optimally coupling the system to the ion source in order to minimize effusiveflow times from the target to the ion source. Therefore, to ensure maximum achievable intensities, RIB sources must be designed so that temperature control can be maintained in the target material reservoir and at the end of the vapor transport tube where the magnitude of the temperature at

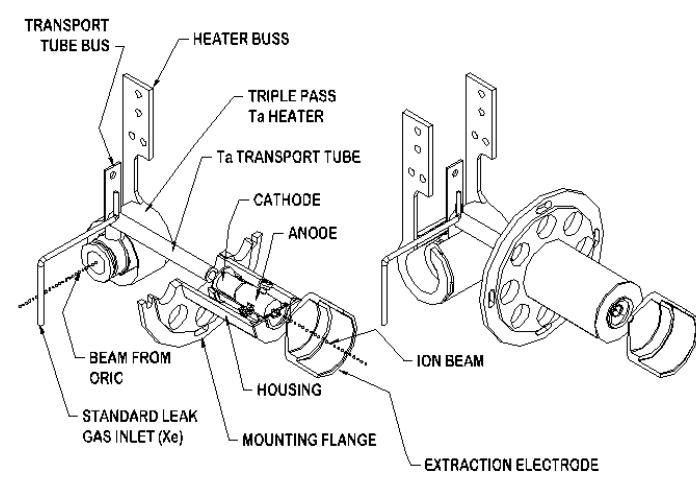

Fig. 1. Isometric of a high-temperature target/ion source used at the HRIBF.

each position may seriously affect source performance.

\subsection{The Electron Beam Plasma Ion Source (EBPIS)}

The Electron Beam Plasma Ion Source (EBPIS), designed and developed for use at the HRIBF, has proved to be a very robust, reliable, versatile, and universal generator of positive-ion beams with many hours recorded for on-line operation. A side-view of the source is displayed in Fig. 2. The source incorporates design features embodied in the on-line sources used at CERNISOLDE [4] and is equipped with a standard Xe leak that serves as a relative monitor of the performance of the source during online operation. The EBPIS utilizes the ionization principles incorporated in the FEBIAD ion source developed by Kirchner, et al. [8-10] in that it does not require a minimum pressure for stable operation (commonly referred to as the Langmuir criterion for stable discharge). It operates at pressures of more than one order of magnitude lower than the Nielsen plasma discharge source [11] as clearly demonstrated by Kirchner and Roeckl [12]. The source is well suited for RIB applications in that it operates stably and efficiently at temperatures $\leq 2000{ }^{\circ} \mathrm{C}$ over a pressure range of $\sim 1 \times 10^{-5}$ to $\sim 2 \times 10^{-4}$ Torr. The emittance (unnormalized), $\varepsilon$, of the source at the $90 \%$ contour has a value, $\varepsilon \cong 25 \pi \mathrm{mm} . \mathrm{mrad}$ at $20 \mathrm{keV}$, ion beam energy.

The efficiency of the source is quite high for slow moving heavy ions; however, for low mass, fast moving atoms with high ionization potentials, the source is not as impressive. For example, the measured ionization efficiencies for the noble gas elements, as reported in Ref. 13, are, respectively, Ne: $1.5 \%$; Ar: $18 \%$; $\mathrm{Kr}: 36 \%$; and Xe: $54 \%$. Based on experiences at the HRIBF, during operation in the more harsh on-line vacuum environment, the efficiencies for $\mathrm{Kr}$ and $\mathrm{Xe}$ are typically considerably less than those reported in Ref. 13 (e.g., 12\% for $\mathrm{Kr}$ and $\sim 20 \%$ for $\mathrm{Xe}$ ). 


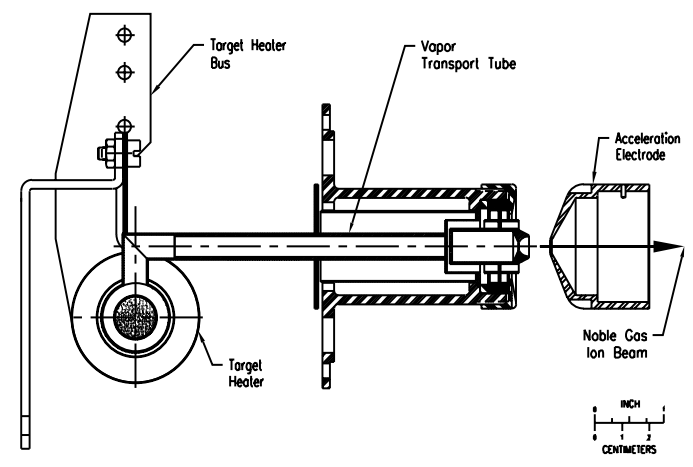

Fig. 2: Side view of the electron beam plasma ion source used for positive RIB generation at the

HRIBF.

\subsection{The Positive-Surface Ionization Source}

An efficient positive-surface ionization source has been designed, fabricated, and initially tested for potential on-line use in generating radioactive ion beams of members of the Group IA elements ( $\mathrm{Li}, \mathrm{Na}, \mathrm{K}, \mathrm{Rb}, \mathrm{Cs}$ and Fr) for the HRIBF research program. The source utilizes direct-surface ionization to form positive-ion beams resulting from collisions of atoms with the surface of a highly porous Ir-coated/carbon-bonded-carbon surface ionizer maintained at $\sim 1600{ }^{\circ} \mathrm{C}$. As is the case for all sources, the target material reservoir is positioned within the inner diameter of a series-connected, resistively-heated, Ta tube designed to reach temperatures exceeding $2000{ }^{0} \mathrm{C}$. The temperature of the vapor transport tube is controlled by changing the current through the tubular structure so that the ionizer is held at nominal temperatures of $\sim 1600{ }^{0} \mathrm{C}$. Group IA species that strike or are evaporated from the hot $\left(1600{ }^{\circ} \mathrm{C}\right)$ Ir are ionized with high efficiency since the work function of the material is high $(\phi: 5.27 \mathrm{eV})$ with respect to the first ionization potentials of these species. The calculated probabilities of these species, $P$, are respectively: Li (Ip : $5.39 \mathrm{eV}): P \sim 0.32$, Na (Ip: $5.14 \mathrm{eV}), P \sim 0.45 ; \mathrm{K}$ (Ip: 4.3 eV), $P \sim 1.0$; Rb (Ip: $4.18 \mathrm{eV}), P \sim 1.0$; Cs Ip: $3.89 \mathrm{eV}), P$ : $\sim 1.0$; Fr $(I p: \sim 3.7 \mathrm{eV}), P: \sim 1.0$. The measured ionization efficiency for a prototype version of the source was $\sim 60$ $90 \%$ for Cs [14]. The estimated emittance (unnormalized), $\varepsilon$, at the $90 \%$ contour has a value, $\varepsilon \sim 15$ $\pi \mathrm{mm} . \mathrm{mrad}$ at $20 \mathrm{keV}$, beam energy.

\subsection{The Kinetic-Ejection Negative-Ion Source}

Chemically active elements are often released from refractory host materials in a variety of molecular forms. Because of the low probability of simultaneously dissociating such molecular carriers and efficiently ionizing their constituents in conventional hot cathode positive ion sources, the species of interest are often distributed in several mass channels in the form of molecular side-band beams that dilute the intensities of the species of interest. Also such carriers typically have low electron affinities and cannot be ionized in traditional negative surface ionization sources. The kinetic-ejection source was conceived [15] to overcome these handicaps, encountered while attempting to generate useable intensifies of ${ }^{17,18} \mathrm{~F}$ for important nuclear astrophysics [1619] and nuclear-structure physics experiments [20]. The source efficiently ionizes atoms or molecules with intermediate to high electron affinities. A side view of the source is shown in Fig. 3. ${ }^{17} \mathrm{~F}$ or ${ }^{18} \mathrm{~F}$ production is effected by irradiation of thin fibrous oxide materials such as $\mathrm{A}_{2} \mathrm{O}_{3} \mathrm{O}_{3} \mathrm{ZrO}_{2}$, or $\mathrm{HfO}_{2}$, held at high temperature in the target reservoir, through the respective reactions ${ }^{16} \mathrm{O}(\mathrm{d}$, $n){ }^{17} \mathrm{~F}$ or ${ }^{18} \mathrm{O}(\mathrm{p}, \mathrm{n}){ }^{18} \mathrm{~F}$. The fluorine isotopes are diffused from the interior of the target material and transported in molecular form (e.g., AlF) through annular apertures that surround the $\mathrm{W}$-ionizer and strike the reduced temperature surface $\left(\sim 400{ }^{0} \mathrm{C}\right)$ of a conically shaped cathode where they are condensed or temporarily reside for a short period of time. Cs vapor, transported from an external oven, is surface ionized by diffusion through a porous $\mathrm{W}$-surface ionizer. An intense beam of $\mathrm{Cs}^{+}$ particles from the ionizer is accelerated to energies of 200 to $300 \mathrm{eV}$ through a Mo grid onto the inner surface of the conical cathode. The beam impacts the surface where it dissociates molecules and ejects their atomic constituents from the surface. Elemental Cs on the surface of the conical cathode lowers the surface work function, essential for effecting negative ionization during the ejection process. Negative ions are extracted through a hole in the apex of the conical surface by the penetrating electrostatic field, maintained between the ground electrode and the cathode. The Mo grid is maintained at a slightly more negative potential than the conical cathode so as to cause a slight drift of ions toward the extraction end of the cone. The positive-Cs ion optics have been designed to effect uniform irradiation of the conical surface to ensure efficient dissociation of molecule and sputter ejection of their atomic constituents from the surface, while the negative-ion extraction optics have been designed to efficiently extract negative ions ejected from the surface. The mass spectrum extracted from the source is simple with essentially $100 \%$ of the $F$ in the

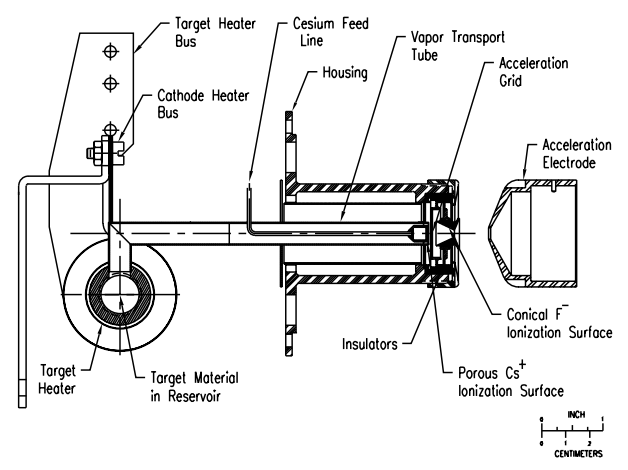

Fig. 3: Side view of the kinetic-ejection negative-ion source (KENIS) used on-line to dissociate molecular carriers of short-lived species such as AlF and negatively ionize their electronegative constituents such as ${ }^{17,18} \mathrm{~F}$. 
atomic mass channel, in contrast to its hot-cathode EBPIS positive counterpart where typically $\sim 13 \%$ of the $F$ resides in the atomic mass channel. According to online measurements, ${ }^{17} \mathrm{~F}$ beam intensities exceed those derived by use of the EBPIS, in combination with charge exchange, by a factor of at least 10. The emittance (unnormalized), $\varepsilon$, of the source has a nominal value of $\varepsilon \cong 48 \pi \mathrm{mm} . \mathrm{mrad}$ at the $80 \%$ contour level at $20-\mathrm{keV}$, beam energy. The source operates very stably and reliably without need for constant operator attention.

\subsection{The Negative-Surface Ionization Source}

An efficient negative-surface ionization source has been designed, fabricated, and tested on-line at the UNISOR facility for use in generating radioactive ion beams of members of the group VIIA elements $(\mathrm{F}, \mathrm{Cl}, \mathrm{Br}$, I, and At) for the HRIBF research program [21]. The source utilizes direct-surface ionization to form negativeion beams from highly electronegative atoms or molecules evaporated from a spherical-sector $\mathrm{LaB}_{6}$ surface ionizer. The salient design features of the source are schematically illustrated in Fig. 4, which shows a cross sectional side view of the target reservoir, the vapor transport tube, ionization region and extraction electrode system of the source. The target material reservoir is heated by means previously described. The transport tube temperature is regulated by varying the current passed along the tube, so that the $\mathrm{LaB}_{6}$ never exceeds $1730{ }^{\circ} \mathrm{C}$ (the thermal dissociation temperature of the material). Typically, the ionizer is operated at $\sim 1650{ }^{0} \mathrm{C}$. Electronegative species that strike and are subsequently evaporated from the spherical geometry $\mathrm{LaB}_{6}$ ionizer surface should be negatively ionized with high probability. For example, the electron affinities, EA, of the Group VIIA halogens (F (EA: $3.4 \mathrm{eV})$; $\mathrm{Cl}$ (EA:3.61 $\mathrm{eV})$; Br (EA: $3.36 \mathrm{eV})$; I (EA: $3.06 \mathrm{eV})$; At (EA: $2.8 \mathrm{eV})$ )

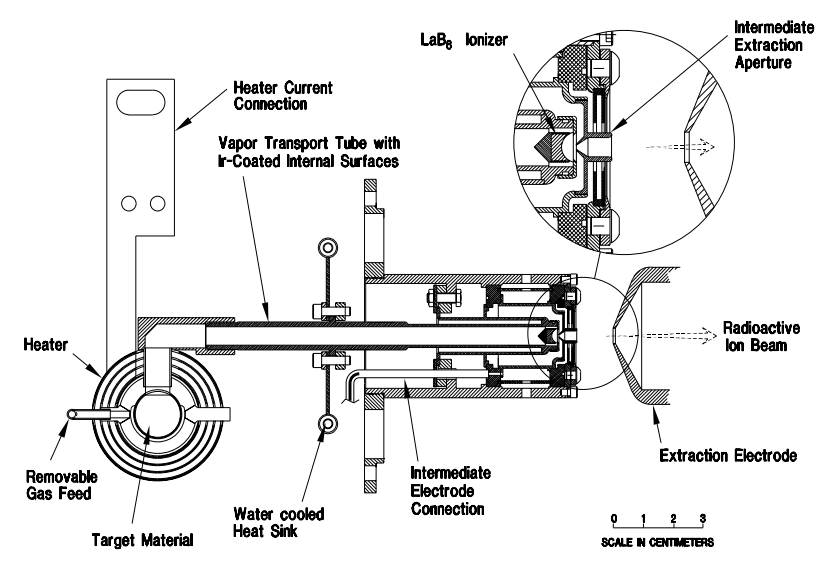

Fig. 4. Side-view of the negative-surface ionization source equipped with a solid, spherical sector $\mathrm{LaB}_{6}$ surface ionizer. species are higher than the work function of $\mathrm{LaB}_{6}(\phi: \sim 2.7$ $\mathrm{eV})$. Negative ions, so produced, are then extracted by applying a positive voltage to the extraction electrode. The measured emittance (unnormalized), $\varepsilon$, of the source is quite low with a nominal value at the $90 \%$ contour of, $\varepsilon \cong 13.7 \pi \mathrm{mm} . \mathrm{mrad}$ at $20 \mathrm{keV}$, beam energy. The results of initial testing indicate that the source is reliable, stable and easy to operate, with nominal efficiencies of $28 \%$ for $\mathrm{Cl}^{-}$and $15 \%$ for $\mathrm{Br}^{-}$beam generation when account is taken of the fractional thermal dissociation of the respective, $\mathrm{AlCl}_{3}$ and $\mathrm{AlBr}_{3}$ carrier molecules used for evaluating the efficiency of the source. The source has been tested on-line for the ionization of short-lived $\mathrm{Br}$ isotopes, produced by irradiating $\mathrm{UC}_{2}$ targets, at the UNISOR facility. The source performed flawlessly and efficiently for many hours of operation.

\subsection{Batch-Mode Cs-Sputter Negative-Ion} Sources

Negative radioactive-ion beams of isotopes with lifetimes in excess of a few hours and electron affinities in excess of $\sim 1 \mathrm{eV}$ can be efficiently generated by use of the Cs-sputter technique. A Cs-sputter negative-ion source has been designed fabricated and extensively tested for the batch-mode generation of RIBs of long-lived species for use in the HRIBF research program [22]. In this scenario, the species of interest are sequentially produced by irradiating target materials with $10-40 \mathrm{MeV}$, light-ion beams from the ORIC for a time sufficiently long for secular equilibrium to transpire (a few half-lives), followed by transfer of the target to a Cs-sputter negativeion source where the irradiated volume is sputtered away by the action of a 1-5 $\mathrm{keV} \mathrm{Cs}{ }^{+}$beam. The $\mathrm{Cs}^{+}$beam not only serves to eject particles from the surface, but also lowers the work function of the surface, thereby greatly enhancing the probability for negative-ion formation since the probability of negative-ion formation depends exponentially on the difference between the electron affinity of the atom and the work function of the surface. RIBs formed by this technique avoid the high target temperatures required for fast diffusion and fast effusiveflow to the ion source. Direct negative-ion formation also avoids losses related to the charge-exchange process required to convert initially positive-ion beams to negative polarity as required for postacceleration with the 25-MV tandem accelerator. Although the source was conceived specifically for the generation of ${ }^{56} \mathrm{Ni}$, it can be employed for the generation of several other long-lived species such as ${ }^{7} \mathrm{Be}$ and ${ }^{18} \mathrm{~F}$. The source is mounted in a vacuum envelope specifically designed for remote installation/removal with the robot controlled radiation handling system used for installing/removing conventional modular high temperature sources routinely used for RIB generation. The source features an eightposition, water-cooled target wheel that can be remotely indexed from the ORIC production position into the Cssputter position. The target wheel can be loaded with 
eight separate targets, thus, providing target variety and the ability to irradiate a second target with beams from ORIC while generating negative-ion beams from an initial target. Beams of $\mathrm{Cs}^{+}$are generated by surface ionization of Cs vapor as it comes in contact with a conical geometry $\mathrm{W}$-surface ionizer. The ionizer/target-electrode system for accelerating $\mathrm{Cs}^{+}$was specifically designed to achieve a large $(\sim 7 \mathrm{~mm})$, uniform irradiation pattern with good overlap with the ORIC production beam $(\sim 6-8 \mathrm{~mm}$ at impact with the target surface) while efficiently extracting negative-ion beams generated in the sputter process. The efficiency for generating ${ }^{56} \mathrm{~N}$ or ${ }^{18} \mathrm{~F}$ is expected to range between $2 \%$ and $5 \%$.

A multi-sample (eight), remotely indexable, Cssputter negative-ion source equipped with a fiber optic remotely indexable target changing mechanism, has also been specifically designed for batch-mode processing of ${ }^{7} \mathrm{Be}$ for future nuclear-astrophysics experiments [23]. The source also utilizes a conical geometry, W-surface ionizer. Sample changing is effected by actuating an electro-pneumatic control system located at ground potential that drives an air-motor driven sample indexing system mounted at high voltage; this arrangement avoids complications associated with indexing mechanisms that rely on electronic power supplies located at high potential. In-beam targets are identified by LED indicator lights derived from fiber optic, Gray-code target-position sensor.

\section{BEAMS DELIVERED FOR RESEARCH}

Highly permeable, thin fibrous $\mathrm{Al}_{2} \mathrm{O}_{3}, \mathrm{ZrO}_{2}, \mathrm{HfO}_{2}$ targets, in combination with the KENIS [15] have enabled research with full stripped beams of ${ }^{17,18} \mathrm{~F}^{9+}$ at intensities up to $3 \times 10^{6} \mathrm{ions} / \mathrm{s}$. The source has performed remarkably well as evidenced by its 1500 hours of on-line use for nuclear astrophysics experiments without failure. Neutron-rich nuclei isotopes are produced in thin-layered composite $\mathrm{UC}_{2}$ targets [24]. More than 132 isotopes from 24 elements have been detected at intensities ranging from $\sim 3 \times 10^{3} / \mathrm{s}$ to $\sim 1 \times 10^{9} / \mathrm{s}$ [25], using the EBPIS $[5,6]$. Several neutron-rich beams have been used in recent months in nuclear structure experiments, including: ${ }^{118} \mathrm{Ag}$ at intensities up to $2 \times 10 \% \mathrm{~s} ;{ }^{126} \mathrm{Sn}$ and ${ }^{132,134} \mathrm{Te}$ at intensities up to $2 \times 10^{7} / \mathrm{s}$. These intensities are consistent with production and release rates of $\geq 1 \times 10^{9}$ /s for these isotopes.

\section{ACKNOWLEDGMENTS}

The author wishes to express his appreciation to students, visiting scientists and all members of the HRIBF staff whose dedicated efforts have made possible implementation of the HRIBF and the successes realized during the past few months. Research at the Oak Ridge National Laboratory is supported by the U.S. Department of Energy under contract DE-AC05-00OR22725 with UT-Battelle, LLC.

\section{REFERENCES}

1. G. D. Alton and J. R. Beene, J. Phys. G: Nucl. Part. Phys. 24 (1998) 1347.

2. G. D. Alton, Proc. 1993 IEEE Particle Accel. Conf. IEEE Cat. No. 93CH3279-7, pp. 2979-2985.

3. Proc. of the Twelfth Int. Conf. on Electromagnetic Isotope Separators and Techniques Related to their Applications, Eds. M. Fujioka, T. Shinozuka, and Y. Kawase, Nucl. Instrum. and Methods B 70 (1992).

4. S. Sundell and H. L. Ravn, Nucl. Instrum. and Methods B 70 (1992) 160.

5. G. D. Alton, D. L. Haynes, G. D. Mills and D. K. Olsen, Nucl. Instrum. and Methods A 328, (1993) 325 .

6. G. D. Alton, Nucl. Instrum. and Methods A $\mathbf{3 8 2}$ (1996) 207.

7. G. D. Alton, J. R. Beene, Y. Liu, Nucl. Instrum. and Methods A 438 (1999) 190.

8. R. Kirchner et al., Nucl. Instrum. and Methods A $\mathbf{2 3 4}$ (1985) 224.

9. R. Kirchner, K. Burkhard, W. Hüller, and 0. Klepper, Nucl. Instrum. and Methods 186 (1981) 295.

10. R. Kirchner, K. Burkhard, and 0. Klepper, Nucl. Instrum. and Methods B 70 (1992) 56.

11. O. Almen and K. 0. Nielsen, Nucl. Instrum. and Methods 1 (1957) 302.

12. R. Kirchner and E. Roeckl, Nucl. Instrum. and Methods 133 (1976) 187.

13. R. Kirchner, Nucl. Instrum. and Methods B $\mathbf{7 0}$ (1992) 186.

14. G. D. Alton, R. F. Welton, B. Cui, S. N. Murray, G. D. Mills, Nucl. Instrum. and Methods B 142 (1998) 578.

15. G. D. Alton, Y. Liu, C. Williams, and S. N. Murray, Nucl. Instrum. and Methods B 170 (2000) 515.

16. D. W. Bardayan et al., Phys. Rev. Lett. 83 (1999) 45.

17. D. W. Bardayan et al., Phys. Rev. C 62 (2000) 042802(R).

18. D. W. Bardayan et al., Phys. Rev. C 63 (2000) 055804.

19. D. W. Bardayan et al., Phys. Rev. C 63 (2001) 065802.

20. J. Gomez del Campo et al., Phys. Rev. Lett. 86 (2001) 43.

21. H. Zaim et al., to be published in AIP Press, Conference Proceedings, 2001; G. D. Alton, et al., (to be published in Nucl. Instrum. and Methods B, 2001).

22. G. D. Mills, G. D. Alton, D. L. Haynes and J. R. Beene, Physics Division Progress Report ORNL69S7, September, 1998.

23. G. D. Alton et al., AIP Press Conf. Proc. CP473, p. 352.

24. G. D. Alton and H. H. Moeller, Physics Division Progress Report ORNL-69S7, September, 1998.

25. D. Stracener et al., to be published in AlP Press, Conference Proceedings, 2001. 